\title{
Removing Partial Blur in a Single Image
}

\author{
Shengyang Dai and Ying Wu \\ EECS Department, Northwestern University, Evanston, IL 60208, USA \\ $\{$ sda690, yingwu\} deecs. northwestern. edu
}

\begin{abstract}
Removing image partial blur is of great practical importance. However, as existing recovery techniques usually assume a one-layer clear image model, they can not characterize the actual generation process of partial blurs. In this paper, a two-layer image model is investigated. Based on the study of partial blur generation process, a novel recovery technique is proposed for a single input image. Both foreground and background layers are recovered simultaneously with the help of the matting technique, powerful image prior models, and user assistance. The effectiveness of the proposed approach is demonstrated by extensive experiments on image recovery and synthesis on real data.
\end{abstract}

\section{Introduction}

Space-invariant image blur has been extensively studied for a long time. However, it is very often in practice that the blur is partial. For example, a fast moving object may be motion blurred in front of a clear background, or the camera may be focused on one object, while the other part of the image is out-of-focus with a large aperture. It is of great practical value to recover such partially blurred images, due to the prevailing uses of current digital cameras.

However, given only one single input image captured by current digital camera, the task of recovering partially blurred image is extremely challenging. Two key issues need to be addressed, i.e., partial blur estimation and partial deblurring. Despite much effort having been made on partial blur estimation, the deblurring part has not been well studied in the literature. This is of great importance in achieving high quality image recovery and editing results. In this paper, we focus on the problem of removing partial blur from one single input image.

The partial deblurring problem is difficult since pixels at the degradation boundary are mixtures of both foreground and background colors at the same positions. Some examples with close-up views are shown in Fig. 1, where we can clearly see how these two components are mingled together. Such degradations can not be characterized by the traditional one-layer model, where the blurred image is modeled as the convolution of a single clear image by a space-

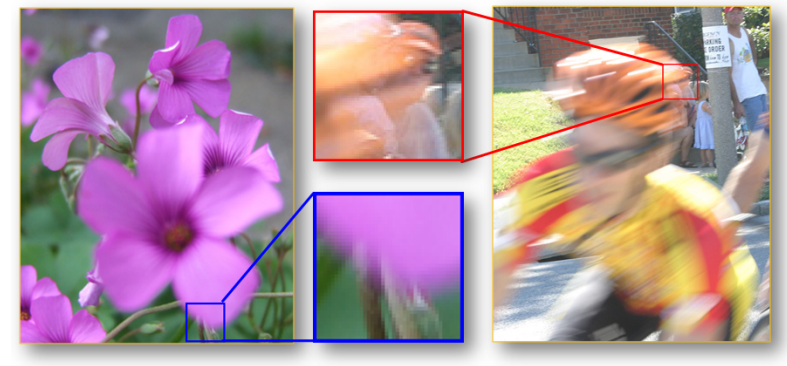

Figure 1. Examples of partially blurred images and close-up views. Pixels at the degradation boundary contain both foreground and background information, which can not be characterized by the traditional one-layer model.

variant PSF (point spread function) [26, 30]. To successfully remove partial image degradations and achieve high quality image recovery, both foreground and background layers have to be recovered simultaneously.

In this paper, to address the above issue, a two-layer image model is investigated. The generation process of partial image degradations based on this two-layer model is studied for the two most common image degradations, i.e., motion blur and out-of-focus blur. Based on this, a novel image recovery scheme is proposed to leverage the recent advances in image prior modeling and matting techniques, with a user assisted initialization step. Due to the precise recovery of image layers and the occlusion mask, high quality image editing applications are enabled. We compare several related techniques, and demonstrate the effectiveness of the proposed approach on various real images.

The related works are summarized in Sec. 2. The generation process is studied in Sec. 3, and our recovery algorithm is introduced in Sec. 4. Experimental results are reported in Sec. 5, and Sec. 6 concludes the paper.

\section{Related work}

Space-invariant blur has been extensively investigated. Recently, more efforts have been taken on the space-variant cases. Two main issues need to be addressed, i.e., blur identification and image recovery.

To estimate motion blurs, most research is focused on the multiple piecewise invariant blur estimation and segmentation problem. For one single input image, this problem is 
approached by [16] based on natural image statistics on image gradients. In [4], a variational and level set technique is used to obtain the boundary between different blurs. The general motion blur problem is studied in [9], where a connection between linear motion blur estimation and optical flow estimation is revealed through a local $\alpha$-motion blur constraint. Out-of-focus blur, in essence, results from the depth, so estimating space-variant focus blurs is closely related to the depth estimation problem [23]. To handle depth discontinuity, an image formation model is studied in $[1,6]$. In [2], local blur parameters are estimated by fitting the edge pattern given a single input, and further used to simulate defocus magnification effect. General non-parametric space-variant blur is studied in [15], based on the sharp edge assumption. Machine learning techniques are employed in [21] to distinguish different kinds of blurs.

To remove partial blur in a single image, a traditional way assumes a one-layer clear image and a space-variant PSF $[4,28]$. This treatment generally has difficulty in handling degradation boundaries. Existing solutions to this issue include minimizing the seam [16], or doing image decomposition before deblurring [27] for rotational blur.

As partial blur removal is extremely difficult for a single input image, researchers also try to make use of multiple images or an image sequence, both for motion blurs [3, 7, 28] and out-of-focus blurs [10, 12, 22]. Besides, efforts have been made to manipulate the image capturing process. The motion blur is studied in $[5,20,24,29]$, and the out-offocus blur in [17, 22, 31]. Despite these efforts, finding effective solutions to remove partial blur in a single input image is still of great interests, given the large quantity of digital photos captured by existing commercial cameras.

\section{Generation model of partial blur}

In this section, based on the two-layer image formation model, the generation process of various partial degradation cases are analyzed, for motion blurs and out-of-focus blurs.

\subsection{The two-layer model for a clear image}

In partially degraded images, pixels at the degradation boundary contain information from both foreground and background at the same position. Such information can not be captured by the traditional one-layer image model, where the degraded image is characterized as the convolution of a single layer clear image and a space-variant PSF. In our work, we use a layered image model to address this issue.

A clear image can be considered as a composition of multiple layers, with an occlusion order. We focus on the case of two layers in this paper, because it is very common and representative in practice. The two-layer technique can be easily generalized to the multiple layer cases. Given the occlusion order, we regard the layer closer to the camera as the clear foreground layer $F$, and the other the clear background layer $B$. One common way to model the clear entire image $I$ is by using a binary mask $m$ as $I=F m+B(1-m)$. However, this model is not precise on the layer boundary, since the pixels there tend to be combinations of both layers due to the foreground transparency and the discretization of the imaging plane. Thus, the combination weight should be soft instead of binary. Based on this, the two-layer image composition model is proposed as:

$$
I=F \alpha+B(1-\alpha),
$$

where $\alpha$ is a clear soft occlusion mask, and $\alpha(x) \in[0,1]$ for each pixel $x$. We regard $F \alpha$ as the clear foreground component, and $B(1-\alpha)$ as the clear background component for the image. This formulation is widely adopted as the image composition equation in alpha matting techniques [18].

Partial image degradations are produced by degrading different image layers in different ways. For example, the foreground may undergo a motion blur on a static background. Or the background may be motion blurred, when the camera is following the moving object, in order to obtain an image of a clear foreground. With a large aperture, the camera may be focused only on one of the image layers, either by the cameraman intentionally or by the auto-focus mechanism in the camera. In this work, we focus on the two common categories of degradation, i.e., motion blur and out-of-focus blur, and discuss the generation process of the partially degraded image $I_{d}$ in different degradation cases. A space-invariant degradation model is assumed within each layer, and characterized by the PSFs $p$ and $q$ for the foreground and background layers, respectively.

\subsection{Motion blur}

Motion blur is a temporal average of clear images taken at different time instants by uniformly sampling the shutter period. We assume the duration of the shutter period is $T$. The foreground layer $F$ moves along a shift function $x_{F}(t)$, where $t \in[0, T]$ is the time. Then at time $t$, the foreground layer is $F\left(x-x_{F}(t)\right)$, and the occlusion mask is $\alpha\left(x-x_{F}(t)\right)$. Thus the foreground component is $F\left(x-x_{F}(t)\right) \alpha\left(x-x_{F}(t)\right)$. Similarly, we assume $x_{B}(t)$ to be the shift function for the background layer $B$, and the background component is $B\left(x-x_{B}(t)\right)\left(1-\alpha\left(x-x_{F}(t)\right)\right)$ at time $t$, due to the occlusion. So the final degraded image $I_{d}$ is the average over time as follows:

$$
\begin{aligned}
I_{d}= & \frac{1}{T} \int_{0}^{T}\left\{F\left(x-x_{F}(t)\right) \alpha\left(x-x_{F}(t)\right)+\right. \\
& \left.B\left(x-x_{B}(t)\right)\left(1-\alpha\left(x-x_{F}(t)\right)\right)\right\} d t \\
= & (F \alpha) * p+B * q \\
& -\frac{1}{T} \int_{0}^{T}\left\{B\left(x-x_{B}(t)\right) \alpha\left(x-x_{F}(t)\right)\right\} d t
\end{aligned}
$$

where $p=\frac{1}{T} \int_{0}^{T} \delta\left(x-x_{F}(t)\right) d t$ is the PSF for the foreground ( $\delta$ is the Dirac delta function), $q=\frac{1}{T} \int_{0}^{T} \delta(x-$ $\left.x_{B}(t)\right) d t$ for the background, and $*$ is the convolution op- 


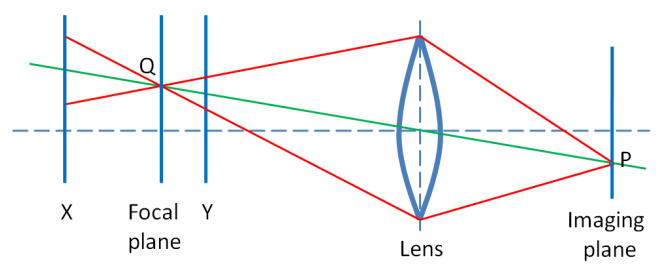

Figure 2. Illustration of the out-of-focus blur generation model.

erator. In the following two typical cases where only one layer is degraded, Eqn. 3 can be further simplified.

Case 1: when the foreground object is moving in front of a static background, we have $x_{B}(t)=0, q=\delta$. Then the resulting degraded image is

$$
I_{d}=(F \alpha) * p+B(1-\alpha * p) .
$$

Case 2: when the background is moving relative to the imaging plane, and with a static foreground, we have $x_{F}(t)=0, p=\delta$. Then the resulting degraded image is

\subsection{Out-of-focus blur}

$$
I_{d}=F \alpha+(B * q)(1-\alpha) \text {. }
$$

The out-of-focus blur can be considered as a spatial average of clear images by sampling the aperture, different from the temporal one for motion blur. According to the reversed projection blurring model [1] with the thin lens model illustrated in Fig. 2, for each point $P$ on the imaging plane, the brightness is the total energy arriving at the lens and passing through the point $Q$ on the focal plane. Although the exact formulation is complicated, similar to the analysis of motion blur, most situations fall into two special cases in practice, i.e., either one of the two layers is in focus.

Case 1: the background layer is in focus, and the foreground layer is out-of-focus (e.g., on plane Y in Fig. 2). Then the contribution of foreground layer is $(F \alpha) * p$, and the occlusion mask for background contribution is $\alpha * p$. So the resulting degraded image is

$$
I_{d}=(F \alpha) * p+B(1-\alpha * p) .
$$

Case 2: the foreground layer is in focus, and the background layer is out-of-focus (e.g., on plane X). Then the foreground contribution is $(F \alpha)$. As the occlusion mask to the background contribution is the same as $\alpha$, the resulting degraded image is

$$
I_{d}=F \alpha+(B * q)(1-\alpha) \text {. }
$$

Similar formulas on the out-of-focus blur generation are discussed in $[1,22]$ as well.

It is interesting to notice that although the motion blur and out-of-focus blur are generated differently, they share the same mathematical form in the above two special cases.

\subsection{Unified formulation of partial blurs}

Although the general cases on two degraded layers are complex, fortunately, the generation model can be much simplified in the cases where only one of the layers is degraded, which are very common in practice. It is easy to verify that they can be unified by the following equation:

$$
I_{d}=(F \alpha) * p+B_{q}\left(1-\alpha_{p}\right),
$$

where $B_{q}=B * q$ is the degraded background layer, and $\alpha_{p}=\alpha * p$ is the degraded occlusion mask. When either the foreground or background layer is not degraded, $p$ or $q$ is the $\delta$ function. Similar form is used in [12] for the general case of out-of-focus blur as an approximation.

On the other hand, once $F, B, \alpha, p$, and $q$ are given, we can also synthesize realistic partial motion or out-of-focus blur effects from Eqn. 8. This synthesis is difficult for the one-layer image model, due to the lack of information at the degradation boundary for both image layers.

\section{Image recovery from partial degradation}

Given the degradation generation process, solving all the unknowns $(F, B, \alpha, p, q)$ in Eqn. 8 is severely under constrained, especially based only on one input image $I_{d}$ as the observation. We exploit a number of effective priors for regularization, including sparsity prior for $F$ and $B$ [17], edge smoothness prior for $\alpha$ [8], and binary prior for $\alpha[14,19]$ (or sharp edge prior [15]). Unfortunately, these prior terms usually lead to non-convex objective functions, which tend to trap the optimization to local minima. Appropriate initialization is critical for searching a better optimum. Thus, we propose a powerful initialization method by leveraging the recent advances in the alpha matting technique.

The entire procedure is illustrated in Fig. 3. To optimize the objective function in Sec. 4.1, we first initialize the value of $\alpha$ (Sec. 4.2) and extract $p q$, then iterate between $F, B$ and $\alpha$ (Sec. 4.3) to obtain the final recovery.

\subsection{The objective function}

We propose to use the following objective function:

$$
\begin{aligned}
O(F, B, \alpha, p, q) & =\left\|(F \alpha) * p+B_{q}\left(1-\alpha_{p}\right)-I_{d}\right\|_{2}^{2} \\
& +R_{\alpha}(\alpha)+R_{i}(F)+R_{i}(B),
\end{aligned}
$$

where $R_{\alpha}(\alpha)$ is the regularization term for $\alpha$, and $R_{i}$ for image layers $F$ and $B$. Besides, we have $\alpha(x) \in[0,1]$ for each pixel $x$ in the image domain $\Omega$.

\section{Regularization for $\alpha$ :}

$$
R_{\alpha}(\alpha)=\lambda_{1}|\alpha|_{\mathcal{G}}+\lambda_{2} \sum_{x \in \Omega}(\gamma(\alpha(x))+\gamma(1-\alpha(x))),
$$

where $|\alpha|_{\mathcal{G}}$ is the Softcuts metric defined in [8] based on a weighted graph $\mathcal{G}$ on the image grid. The Softcuts metric approximates the average length of the image level lines. Thus adding it as a regularization term helps obtain smooth soft edges with transitions. The underlying assumption is that the foreground object tends to have a smooth soft matte, which is valid for most real world objects. In the second part of Eqn. 10, $\gamma(t)=|t|^{0.8}$ is used to favor binary $\alpha$ values [19], since $(\gamma(\alpha)+\gamma(1-\alpha))$ reaches its minimum when $\alpha \in\{0,1\}$. The assumption made here is that clear images usually have sharp edges, which is generally hold in practice. We use $\lambda_{1}=\lambda_{2}=0.01$ in our experiments as the combination weights. 


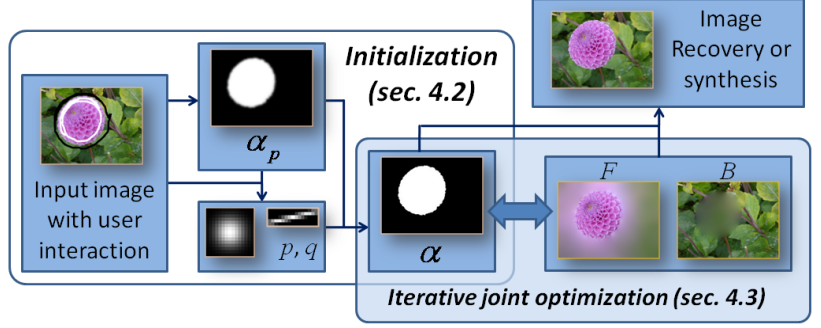

Figure 3. Overview of our partial image recovery algorithm.

\section{Regularization for $F$ and $B$}

$$
R_{i}(I)=\lambda_{3} \sum_{\{x, y\} \in \mathcal{N}} \rho(I(x)-I(y)),
$$

where $I \in\{F, B\}$, and the summation is over the set $\mathcal{N}$ of all neighboring pixel pairs. We use $\rho(t)=|t|^{0.9}$ as the sparsity prior term which prefers sparse image gradient [17], and set $\lambda_{3}=0.005$ in our experiments as the weight.

\subsection{Initialization}

Initialization is critical for optimizing non-convex objective functions. As illustrated in Fig. 3, we first extract the degraded occlusion mask $\alpha_{p}$ by using a matting technique. After that, the degradation kernels $p$ and $q$ are estimated by analyzing both $I_{d}$ and $\alpha_{p}$, and the non-blurred occlusion mask $\alpha$ is recovered afterwards.

Estimating $\alpha_{p}$ : By assuming a locally smooth foreground $F$ and denoting $F_{p}=F * p$, we have $(F \alpha) * p \simeq$ $F_{p} \alpha_{p}$ (it is exact for the 2 nd cases in both kinds of blur in Sec. 3, where $p=\delta$ ). Putting it in Eqn. 8, we have

$$
I_{d} \simeq F_{p} \alpha_{p}+B_{q}\left(1-\alpha_{p}\right) .
$$

As the RHS of Eqn 12 shares the same mathematical form as in the composition equation in alpha matting (Eqn. 1), existing graphics techniques can provide powerful algorithms to solve $\alpha_{p}$ from $I_{d}$ [18]. The reason they can be applied here is that both $F_{p}$ and $B_{q}$ can be assumed locally smooth, which is consistent with the assumption made in matting techniques. A major benefit of using matting techniques is that color information from all three color channels is implicitly integrated (e.g., the linear color model in [18]), and provides powerful regularization terms for the decomposition. Such information is usually ignored in methods processing each color channel separately.

The closed-form solution [18] to the alpha channel is adopted in our work to initialize $\alpha_{p}$ by using moderate user inputs that indicate some pure foreground and background pixels. Possible ways to automate this process are discussed in Sec. 4.4. An example is shown in Fig. 4, where (b) visualizes the user strokes, and (c) visualizes the extracted degraded occlusion mask $\alpha_{p}$.

Estimating $p, q$ : Once having $\alpha_{p}$, pure foreground and background regions can be identified. By applying existing space-invariant estimation algorithms on these regions, we can obtain their PSFs (i.e., $p$ and $q$ ). In our work, we

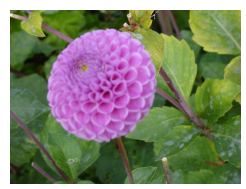

(a)

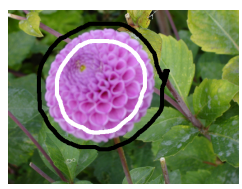

(b)

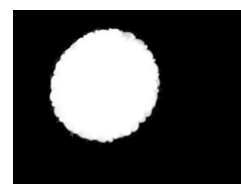

(c)
Figure 4. Illustration of using user interaction for extracting $\alpha_{p}$, (a) the input image, (b) user indication of the pure foreground (white) and background (black) pixels, and (c) extracted $\alpha_{p}$.

use the methods in [9] and [2] to estimate the motion blur and the out-of-focus blur, respectively. Notice that in [9], the $\alpha$-motion blur constraint can be applied directly on $\alpha_{p}$. The estimation of $p$ and $q$ is of good accuracy once having the rough separation of the foreground and the background, thus they are fixed in the following steps.

Estimating $\alpha$ : Given $\alpha_{p}$ and $p$, estimating $\alpha$ is a standard deconvolution problem. But it becomes non-trivial here, because the extracted $\alpha_{p}$ is usually very noisy. The reason is that Eqn. 12 is an approximation, so that the matting algorithm does not always give perfect result. This complication greatly limits the applicability of current deconvolution algorithms and blind $\alpha$ restoration method as in [14]. To address this issue, we use $R_{\alpha}$ defined in Eqn.10 for a regularization in the following objective function

$$
\left\|\alpha * p-\alpha_{p}\right\|_{2}^{2}+R_{\alpha}(\alpha)
$$

Gradient decent is used for optimization, where it is easy to enforce the range constraint $\alpha \in[0,1]$ by truncating the value in each iteration. The initial estimation of $\alpha$ is set to be $\alpha_{p}$. Large weights $\left(\lambda_{1}=0.20\right.$ and $\left.\lambda_{2}=0.02\right)$ are used to alleviate the noise in this step. For the 2nd cases in both kinds of blur, this step is not necessary, since $\alpha=\alpha_{p}$.

An example is shown in Fig.5. The extracted $\alpha_{p}$ is shown in (a), which is very noisy. Results of some existing deconvolution algorithms are shown in (b)(c), leading to either jaggy or blurry $\alpha$. By using the proposed method, smooth and nearly binary $\alpha$ is obtained. The effectiveness of the edge smoothness prior and the binary prior is demonstrated by comparing Fig. 5(f) with (d) and (e), respectively.

\subsection{Recovering $(F, B, \alpha)$}

Finally, we iterate between the image layers $(F, B)$ and the occlusion mask $\alpha$ to obtain the final recovery.

Updating $F$ and $B$ given $\alpha$ : the iterative re-weighted least square (IRLS) method [17] is applied to optimize the non-convex function in Eqn. 9 by fixing $\alpha$. At each step of IRLS, we need to optimize the following quadratic form, with a matrix notation as follows:

$$
\left\|P x_{F}+Q x_{B}-y\right\|_{2}^{2}+\lambda_{3}\left(\left\|D_{F} x_{F}\right\|_{2}^{2}+\left\|D_{B} x_{B}\right\|_{2}^{2}\right) \text {, }
$$
where $x_{F}, x_{B}$, and $y$ are the vector representations of $F$, $B$, and $I_{b} . P$ is the transformation matrix that achieves the operation of element-wise multiplication of $\alpha$, followed by the convolution operation with $p$, and $Q$ for convolution operation with $q$ followed by element-wise multiplication of $\left(1-\alpha_{p}\right) . D_{F}$ and $D_{B}$ account for the weighted gradient 


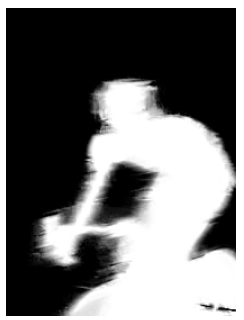

(a)

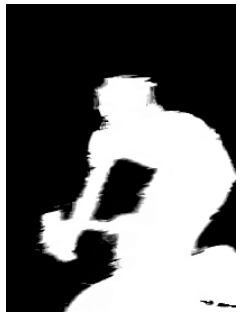

(d)

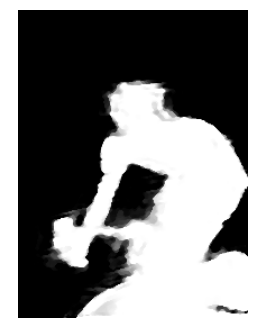

(b)

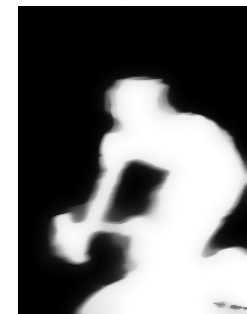

(e)

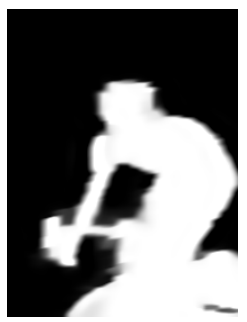

(c)

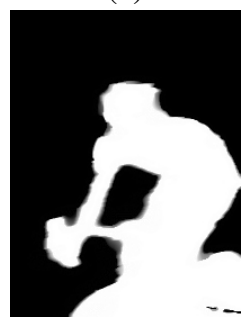

(f)
Figure 5. Comparison of $\alpha$ estimation algorithms given $\alpha_{p}$ and $p$ (the estimated motion vector is $\left.(14,-3)^{\prime}\right)$, (a) noisy $\alpha_{p}$ extracted by the matting algorithm, (b) result by the back-projection method [13], (c) result by deconvolution with sparsity prior [17], (d) our result without the edge smoothness prior term, (e) our result without the binary prior term, (f) result by our method (Eqn. 13).

filter for $R_{i}$ based on the IRLS method. Equation 14 is optimized by solving a sparse linear system $A x=b$, where

$$
\begin{gathered}
A=\left[\begin{array}{ll}
P^{\prime} P & P^{\prime} Q \\
Q^{\prime} P & Q^{\prime} Q
\end{array}\right]+\lambda\left[\begin{array}{cc}
D_{F}^{\prime} D_{F} & 0 \\
0 & D_{B}^{\prime} D_{B}
\end{array}\right] \\
x=\left[\begin{array}{l}
x_{F} \\
x_{B}
\end{array}\right], b=\left[\begin{array}{l}
P^{\prime} y \\
Q^{\prime} y
\end{array}\right] .
\end{gathered}
$$

The conjugate gradient method is used for optimization. Color channels are processed independently. Both $F$ and $B$ are initialized to be $I_{d}$.

Updating $\alpha$ given $F$ and $B$ : from Eqn. 9, we optimize

$$
\left\|I_{d}-(F \alpha) * p-B_{q}\left(1-\alpha_{p}\right)\right\|_{2}^{2}+R_{\alpha}(\alpha) \text {. }
$$

Similar to Sec. 4.2, the gradient decent method is applied, and the range constraint is enforced during the iteration.

\subsection{Discussions}

Different from solving both foreground and background jointly as in our method, another way is to use the matting technique not only to solve $\alpha_{p}$, but separate the foreground and background components as well, and recover them independently afterwards. This scheme is suggested in [27] when applying their rotational deblur algorithm on partial degradation. However, such a scheme tends to create artifacts since the degradation model is ignored in the decomposition step. An illustrative example is given in Fig. 6, where the input image (a) is generated by a motion blurred foreground (c) and a still background (d). The matting algorithm gives the decomposition as in (e) and (f) with apparent artifacts, which brings difficulty in both image recovery and synthesis. The proposed algorithm combines the decomposition together with the degradation model. It leads to accurate estimation as shown in (g) and (h).

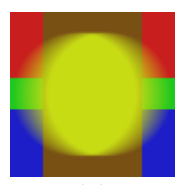

(a)

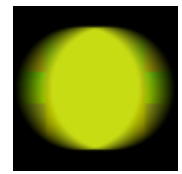

(e)

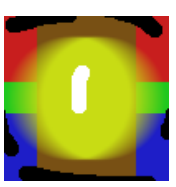

(b)

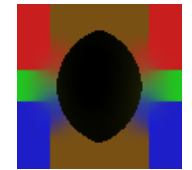

(f)

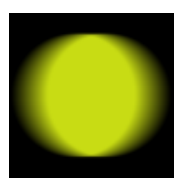

(c)

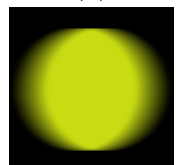

(g)

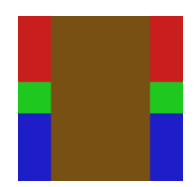

(d)

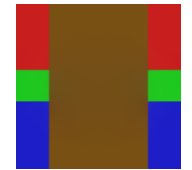

(h)
Figure 6. Result comparison of estimating $F$ and $B$ with different approaches, (a) the input image $I_{d}$, (b) user interaction, (c)(d) are the ground truth for the blurred foreground component $((F \alpha) * p)$ and the clear background $B$, (e)(f) are the estimation results by matting algorithm [18], (g)(h) are our estimation results.

Currently, we rely on moderate user interaction to provide rough cues for initialization. In fact, user interaction is widely used in challenging image recovery tasks [11, 27, 28]. An important and parallel research direction is how to use local estimation techniques to fully automate this process. Much attention has been paid on that direction recently, for both motion blur and out-of-focus blur $[2,9,15,16,21]$. However, to obtain high quality recovery results, the local estimation algorithms need to distinguish the pure foreground, the pure background, and the degradation boundary region with high precision. This is extremely hard, especially for the cases that are ambiguous if only low level local information is considered, even with the region smoothing techniques (e.g., Markov Random Fields). The ultimate solution to this problem may involve higher level information, such as object level segmentation, occlusion reasoning, and inner edge and depth discontinuity edge discrimination, which can be easily provided by users on the other hand. An alternative is to use local estimation techniques to facilitate the user interaction.

In terms of image recovery for out-of-focus blur, there are some closely related techniques in the literature, such as the foreground matte extraction algorithm in [22] and the high dynamic range image recovery algorithm in [12]. The major difference is that they make use of multiple input images, and can not be applied on motion blur.

\section{Experiments}

We demonstrate the effectiveness of the proposed approach by extensive comparison experiments, and applications to image synthesis over real data.

Figure 7 shows our result on a real image with motion blurred foreground. This is a very difficult case. Because of the motion blur with large extend, a large number of pixels close to the degradation boundary are mixtures of both foreground and background colors. Our recovery result is shown in (b), where the image details are successfully recovered, such as regions on the cloth and arms. In the mean 


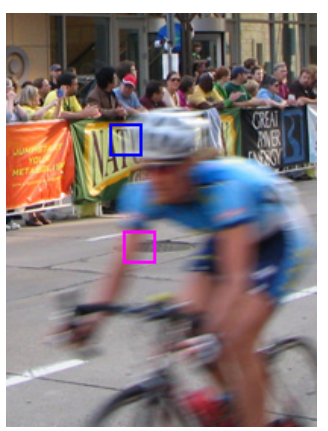

(a) input image $I_{d}$

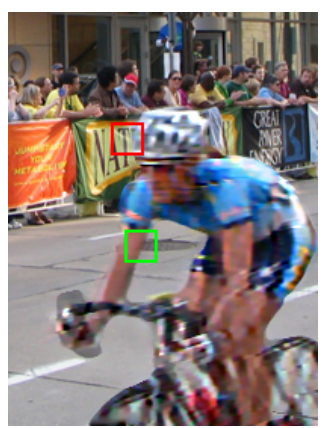

(b) recovery result $I$

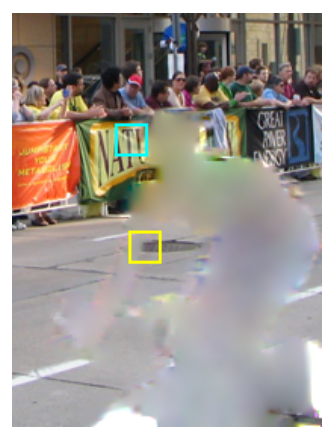

(c) recovered $B$

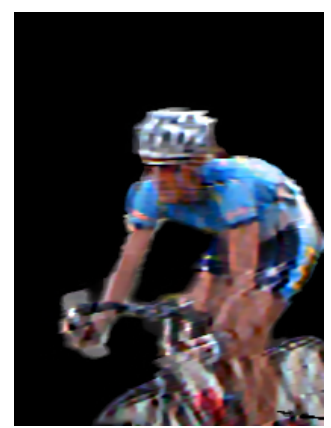

(d) recovered $F \alpha$

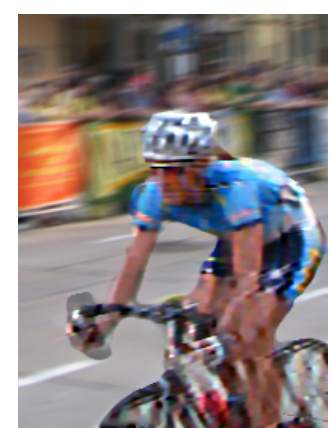

(e) synthetic result
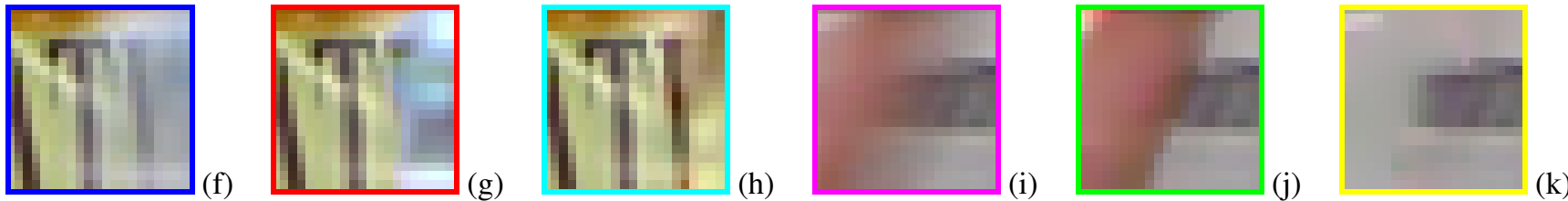

Figure 7. Our recovery and synthesis result from partially motion blurred image. (f)-(k) are close-up views of patches in (a)-(c), where (f)(i) are from $(\mathrm{a}),(\mathrm{g})(\mathrm{j})$ are from $(\mathrm{b})$, and $(\mathrm{h})(\mathrm{k})$ are from $(\mathrm{c})$, notice that the foreground and background components are correctly separated.

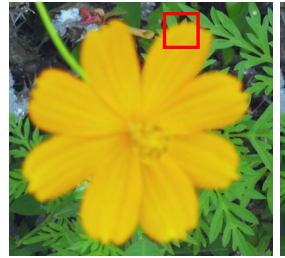

(a)

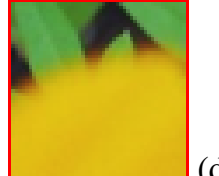

(d)

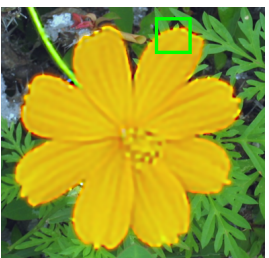

(b)

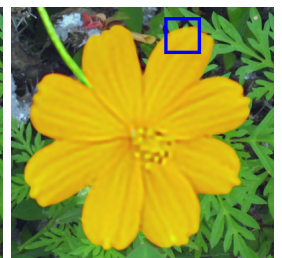

(c)

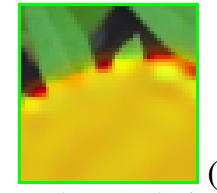

(e)

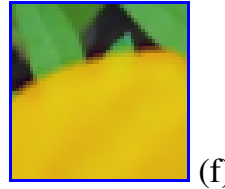

(f)

Figure 8. Comparison to deconvolution algorithm based on the one-layer image model and space-variant PSF, (a) input image, (b) deconvolution result by the one-layer model, (c) deconvolution result by the proposed two-layer model, (d), (e), and (f) are close-up views of patches from (a), (b), and (c), respectively. Our method gives recovery result of much higher quality.

while, the background is also correctly separated (in (c)). Close-up views are highlighted in (f)-(k), where we can clearly see the successful separation of image layers. The extraction of both $F$ and $B$ enables image editing application, such as synthesizing the effect of camera tracing the foreground, shown in (e).

Figure 8 compares the proposed method to the traditional way of doing space-variant deconvolution that assumes a one-layer model for the clear image. The same space-variant kernel is used for both methods. As shown in (b) and (e), the one-layer model tends to generate artifacts at the degradation boundary. That is because it is unable to characterize the image generation process correctly.

In Fig. 9 and Fig. 10, we compare our algorithm to the existing automatic partial blur estimation/recovery techniques. Since it is extremely difficult for automatic algorithms to achieve local estimation with high precision, their

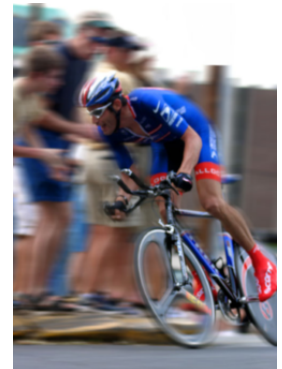

(a) input image

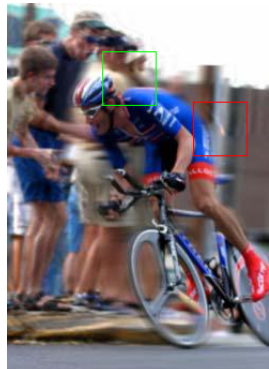

(b) result in [16]

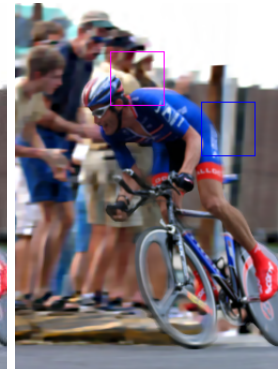

(c) our result
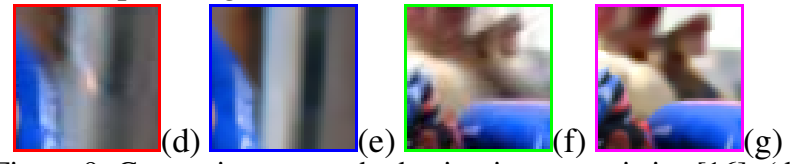

Figure 9. Comparison to method using image statistics [16], (d) (g) are close-up views ((d)(f) are from (b), and (e)(g) are from (c)).

results contain salient artifacts, which are corrected with the help of moderate user interactions. In Fig. 9, comparing with [16], our method obtains better recovery result. The advantage of the proposed technique is clearly shown at the background regions close to the object. In Fig. 10, we compare our result to the defocus magnification method [2]. Our method not only enables deblurring shown in (b), but avoids over blurring the foreground during the synthesis as well.

Figure 11 demonstrates the application of the proposed algorithm to simultaneous image recovery and inpainting. In (a), an out-of-focus blurred background image is occluded by a clear foreground. Due to the extent of the blur kernel, non-occluded background pixels actually contain color information for some of the occluded pixels of the clear background layer. Besides, although the foreground is in focus, pixels on the boundary between foreground and background are still mixtures of the both. The proposed approach makes use of all these information to recover the clear background as shown in (c). Such a "seeing beyond 


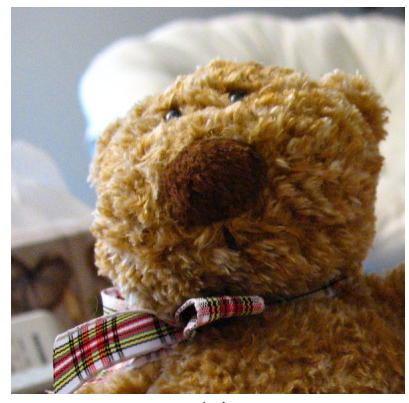

(a)

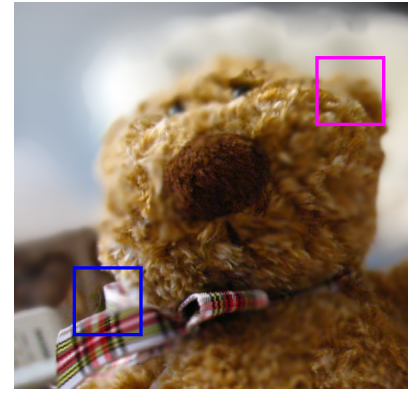

(c)
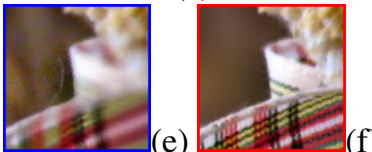

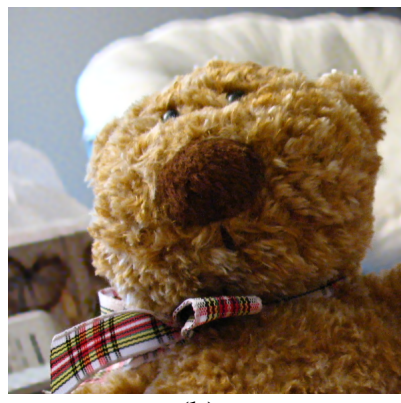

(b)

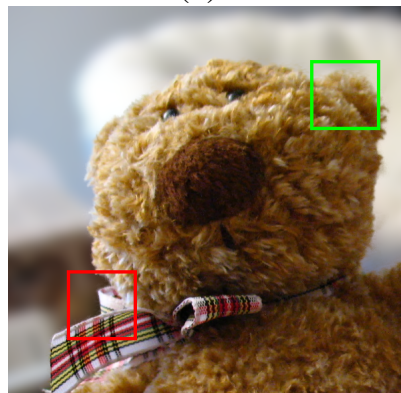

(d)
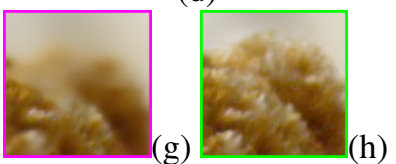

Figure 10. Comparison to defocus magnification [2], (a) input image, (b) our recovery result, (c) synthesis result of defocus magnification [2], (d) our synthesis result, (e)-(h) are close-up views $((\mathrm{e})(\mathrm{g})$ are from $(\mathrm{c}),(\mathrm{f})(\mathrm{h})$ are from $(\mathrm{d}))$. Foreground image details are kept at the degradation boundary by our method.

occlusions" [10] mechanism produces more visually pleasant result than the state-of-the-art image inpainting technique [25], which only use an image prior model and requires more precise user indication as in (e).

More results on real images are shown in Fig. 12. Various cases of partial degradation are included. By extracting both image layers, realistic synthesis results are obtained based on the generation model in Eqn. 8. The moderate user interactions used in our work are shown in Fig. 13.

In practice, we found the algorithm is insensitive to parameters. However, since relatively strong prior terms are applied due to the severely underdetermined natural of the problem, some artifacts can be observed, such as oversmoothness in Fig. 9. This issue could be potentially addressed by using spatially adaptive weights. The algorithm may fail when the prior itself does not hold, e.g., the edge smoothness assumption [8] is invalid for hairy boundaries. Besides, only two-layer model is currently considered, with one of the layered blurred in a spatially invariant way.

\section{Conclusion}

In this paper, a novel algorithm is proposed for removing partial blur from a single image input. This new method is based on the study of the generation process on a two-

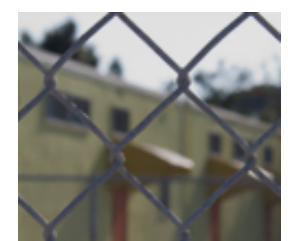

(a)

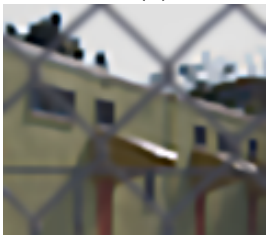

(d)

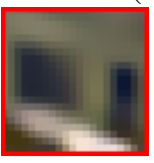

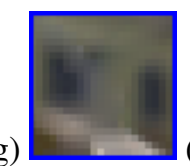

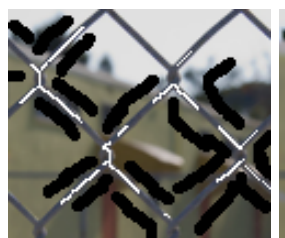

(b)

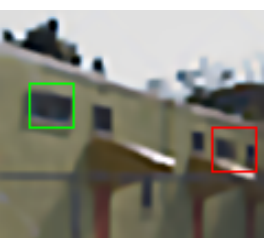

(c)
Figure 11. Comparison to image inpainting techniques, (a) input image with clear foreground and out-of-focus blurred background, (b) user interaction used in our method, (c) our background recovery result, (d) our synthesis result for the focus changing effect, (e) user interaction for applying the inpainting method [25], (f) inpainting result with the Fields of Experts model [25]. (g)-(j) are close-up views ((g)(i) are from (c), (h)(j) are from (f)).

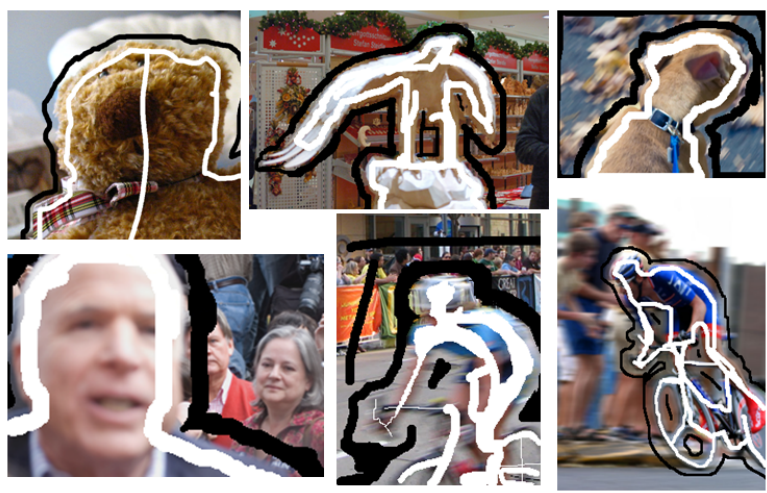

Figure 13. User interaction used for obtaining our results.

layer image model. Both foreground and background layers are recovered simultaneously, which enables high quality recovery and synthesis for real images. Future research includes the robust integration of the local estimation techniques towards a fully automatic process of image recovery.

\section{Acknowledgement}

This work was supported in part by National Science Foundation grant IIS-0347877 and US Army Research Laboratory and the US Army Research Office under grant ARO W911NF-08-1-0504. We thank the anonymous reviewers for their constructive comments.

\section{References}

[1] N. Asada, H. Fujiwara, and T. Matsuyama. Seeing behind the scene: analysis of photometric properties of occluding edges by the reversed projection blurring model. IEEE Trans. on PAMI, 1998. 

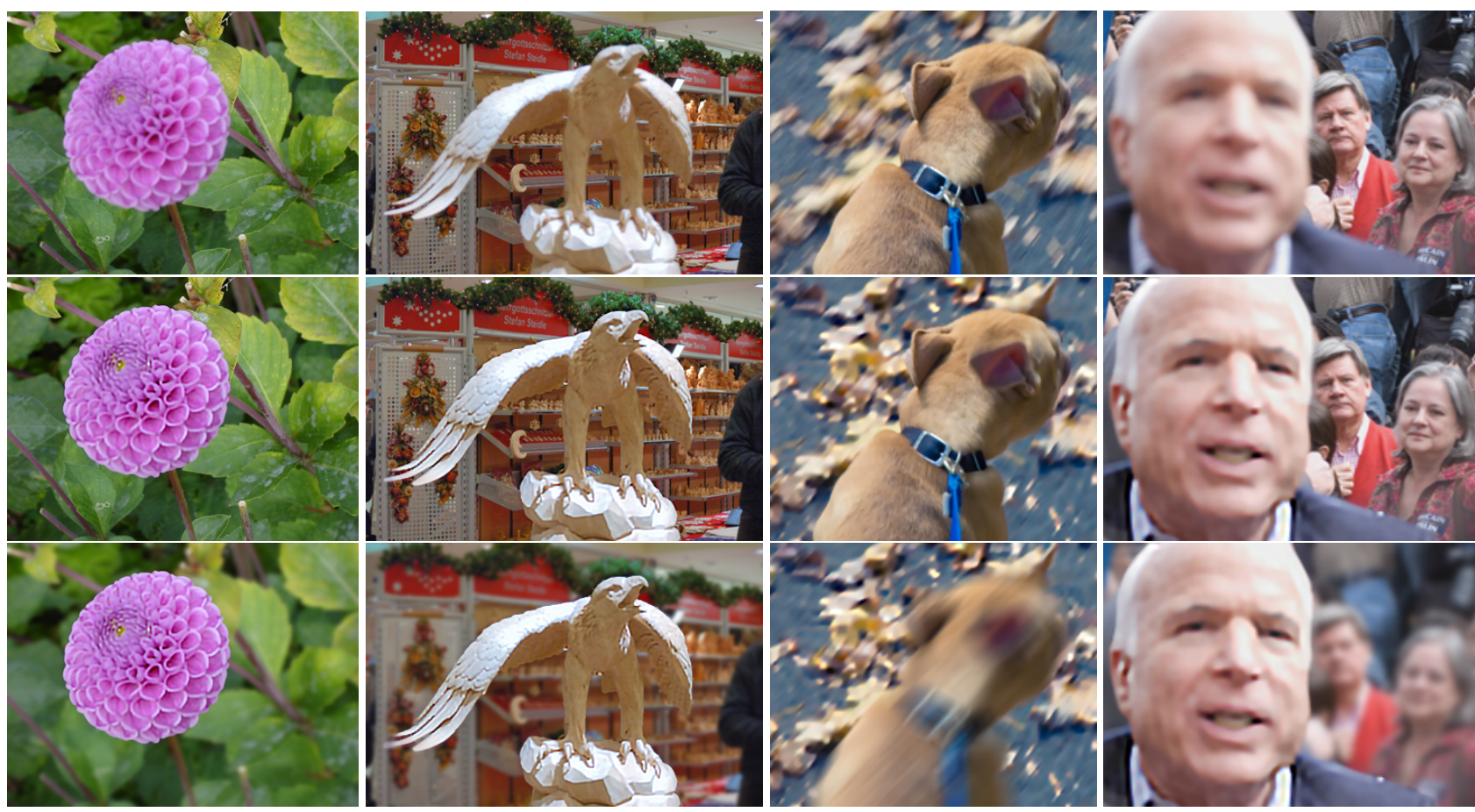

Figure 12. Additional results, 1st row: input partially blurred images, 2nd row: recovery results, 3rd row: partial blur synthesis results.

[2] S. Bae and F. Durand. Defocus magnification. In Eurographics, 2007.

[3] L. Bar, B. Berkels, M. Rumpf, and G. Sapiro. A variational framework for simultaneous motion estimation and restoration of motionblurred video. In ICCV, 2007.

[4] L. Bar, N. Sochen, and N. Kiryati. Restoration of images with piecewise space-variant blur. In SSVM, 2007.

[5] M. Ben-Ezra and S. K. Nayar. Motion-based motion deblurring. IEEE Trans. on PAMI, 2004.

[6] S. S. Bhasin and S. Chaudhuri. Depth from defocus in presence of partial self occlusion. In ICCV, 2001.

[7] S. Cho, Y. Matsushita, and S. Lee. Removing non-uniform motion blur from images. In ICCV, 2007.

[8] S. Dai, M. Han, W. Xu, Y. Wu, and Y. Gong. Soft edge smoothness prior for alpha channel super resolution. In CVPR, 2007.

[9] S. Dai and Y. Wu. Motion from blur. In $C V P R, 2008$.

[10] P. Favaro and S. Soatto. Seeing beyond occlusions (and other marvels of a finite lens aperture). In CVPR, 2003.

[11] R. Fergus, B. Singh, A. Hertzmann, S. T. Roweis, and W. T. Freeman. Removing camera shake from a single photograph. ACM Trans. on Graphics (Proc. SIGGRAPH), 2006.

[12] S. W. Hasinoff and K. N. Kutulakos. A layer-based restoration framework for variable-aperture photography. In ICCV, 2007.

[13] M. Irani and S. Peleg. Motion analysis for image enhancement: resolution, occlusion and transparency. JVCIP, 1993.

[14] J. Jia. Single image motion deblurring using transparency. In $C V P R$, 2007.

[15] N. Joshi, R. Szeliski, and D. J. Kriegman. Psf estimation using sharp edge prediction. In $C V P R, 2008$.

[16] A. Levin. Blind motion deblurring using image statistics. In NIPS, 2006.

[17] A. Levin, R. Fergus, F. Durand, and W. T. Freeman. Image and depth from a conventional camera with a coded aperture. ACM Trans. on Graphics (Proc. SIGGRAPH), 2007.

[18] A. Levin, D. Lischinski, and Y. Weiss. A closed form solution to natural image matting. IEEE Trans. on PAMI, 2007.
[19] A. Levin, A. Rav-Acha, and D. Lischinski. Spectral matting. In CVPR, 2007

[20] A. Levin, P. Sand, T. S. Cho, F. Durand, and W. T. Freeman. Motioninvariant photography. ACM Trans. on Graphics (Proc. SIGGRAPH), 2008.

[21] R. Liu, Z. Li, and J. Jia. Image partial blur detection and classification. In $C V P R, 2008$

[22] M. McGuire, W. Matusik, H. Pfister, J. F. Hughes, and F. Durand. Defocus video matting. ACM Trans. on Graphics (Proc. SIGGRAPH), 2005.

[23] A. P. Pentland. A new sense for depth of field. IEEE Trans. on PAMI, 1987.

[24] R. Raskar, A. Agrawal, and J. Tumblin. Coded exposure photography: Motion deblurring using fluttered shutter. ACM Trans. on Graphics (Proc. SIGGRAPH), 2006.

[25] S. Roth and M. J. Black. Fields of experts: A framework for learning image priors. In $C V P R, 2005$.

[26] A. A. Sawchuk. Space-variant image motion degradation and restoration. Proceedings of the IEEE, 1972.

[27] Q. Shan, W. Xiong, and J. Jia. Rotational motion deblurring of a rigid object from a single image. In $I C C V, 2007$.

[28] M. Sorel and J. Flusser. Space-variant restoration of images degraded by camera motion blur. IEEE Trans. on Image Processing, 2008.

[29] Y.-W. Tai, H. Du, M. S. Brown, and S. Lin. Image/video deblurring using a hybrid camera. In CVPR, 2008.

[30] H. J. Trussell and B. R. Hunt. Image restoration of space-variant blurs by sectional methods. IEEE Trans. on Acoust. Speech, Signal Processing, 1978.

[31] A. Veeraraghavan, R. Raskar, A. Agrawal, A. Mohan, and J. Tumblin. Dappled photography: Mask enhanced cameras for heterodyned light fields and coded aperture refocusing. ACM Trans. on Graphics (Proc. SIGGRAPH), 2007. 